\section{En face OCT angiography demonstrates flow in early type 3 neovascularization (retinal angiomatous proliferation)}

KK Dansingani $i^{1,2,3}$, J Naysan ${ }^{1,2,4}$ and KB Freund ${ }^{1,2,4}$

\begin{abstract}
Introduction The characteristics of type 3 neovascularization (NV), also known as retinal angiomatous proliferation, have been well described clinically, as well as with fluorescein angiography (FA), indocyanine green angiography, and optical coherence tomography (OCT). OCT angiography (OCT-

hyperfluorescent focus that typically appears first during dye transit, followed by late leakage. Optical coherence tomography (OCT) localizes the lesion to the deeper layers of the retina.

The advent of OCT angiography (OCT-A) enables retinal microvascular flow to be imaged in the clinical setting without the injection of intravenous fluorescent dye.
\end{abstract} A) is a novel and non-invasive technique for imaging retinal microvasculature by detecting changes, with respect to time, in reflectivity related to blood flow.

Method In this case series, we describe two patients who presented with type $3 \mathrm{NV}$ and underwent clinical examination and multimodal imaging, including OCT-A.

Results In the first patient, OCT-A demonstrated flow within two separate lesions in the same eye, one of which was only weakly detected by FA. In the second patient, sequential OCT-A demonstrated a reduction in intralesional flow following intravitreal therapy. Conclusions OCT-A may have a role in the early diagnosis of type $3 \mathrm{NV}$ and in assessing the response to treatment. Further studies are needed to determine sensitivity and specificity.

Eye (2015) 29, 703-706; doi:10.1038/eye.2015.27; published online 6 March 2015

\section{Introduction}

Type 3 neovascularization (NV), also known as retinal angiomatous proliferation, is thought to arise predominantly from the deep capillary plexus of the retina and grow toward the RPE, forming anastomoses with type 1 (sub-RPE) NV. ${ }^{1-4}$ Fluorescein angiography (FA) reveals, at the site of the intraretinal vascular lesion, a

\section{Case report 1}

An 88-year-old female with a 3-year history of NV age-related macular degeneration (NV-AMD) in her right eye presented for a maintenance injection of intravitreal bevacizumab. Fundus examination showed a small new intraretinal hemorrhage in her left eye. Spectral domain OCT (SD-OCT; OCT +HRA, Heidelberg Engineering) and FA confirmed the presence of two separate type 3 NV lesions (Figures 1a and e), the more superior lesion being only weakly detectable on FA.

The patient also underwent OCT-A (Avanti, Optovue, Fremont, CA, USA), demonstrating flow within the active lesions (Figures $1 \mathrm{f}$ and $\mathrm{g}$ ). Intravitreal ranibizumab was administered to the left eye to initiate a course of therapy.

\section{Case report 2}

A 70-year-old female with a prior history of type $3 \mathrm{NV}$ in her left eye presented for follow up. Assessment of the previously non-NV right eye confirmed a new type $3 \mathrm{NV}$ lesion as documented by color photography, FA/ICG, and SD-OCT (Figures 2a and d). The patient underwent swept-source OCT and OCT-A (Topcon DRI OCT-1, Topcon Corporation, Tokyo, Japan), following which the volume data
${ }^{1}$ Vitreous Retina Macula Consultants of New York, New York, NY, USA

${ }^{2}$ LuEsther T. Mertz Retinal Research Center, Manhattan Eye, Ear and Throat Hospital, New York, NY, USA

${ }^{3}$ Moorfields Eye Hospital, London, UK

${ }^{4}$ Department of Ophthalmology, New York University School of Medicine, New York, NY, USA

Correspondence: KB Freund, Vitreous Retina Macula Consultants of New York, 460 Park Avenue, 5th Floor, New York, NY 10022, USA Tel: +1 212861 9797; Fax: +1 2126280698 . E-mail: kbfnyf@aol.com

Received: 1 December 2014 Accepted in revised form: 28 January 2015 Published online: 6 March 2015 


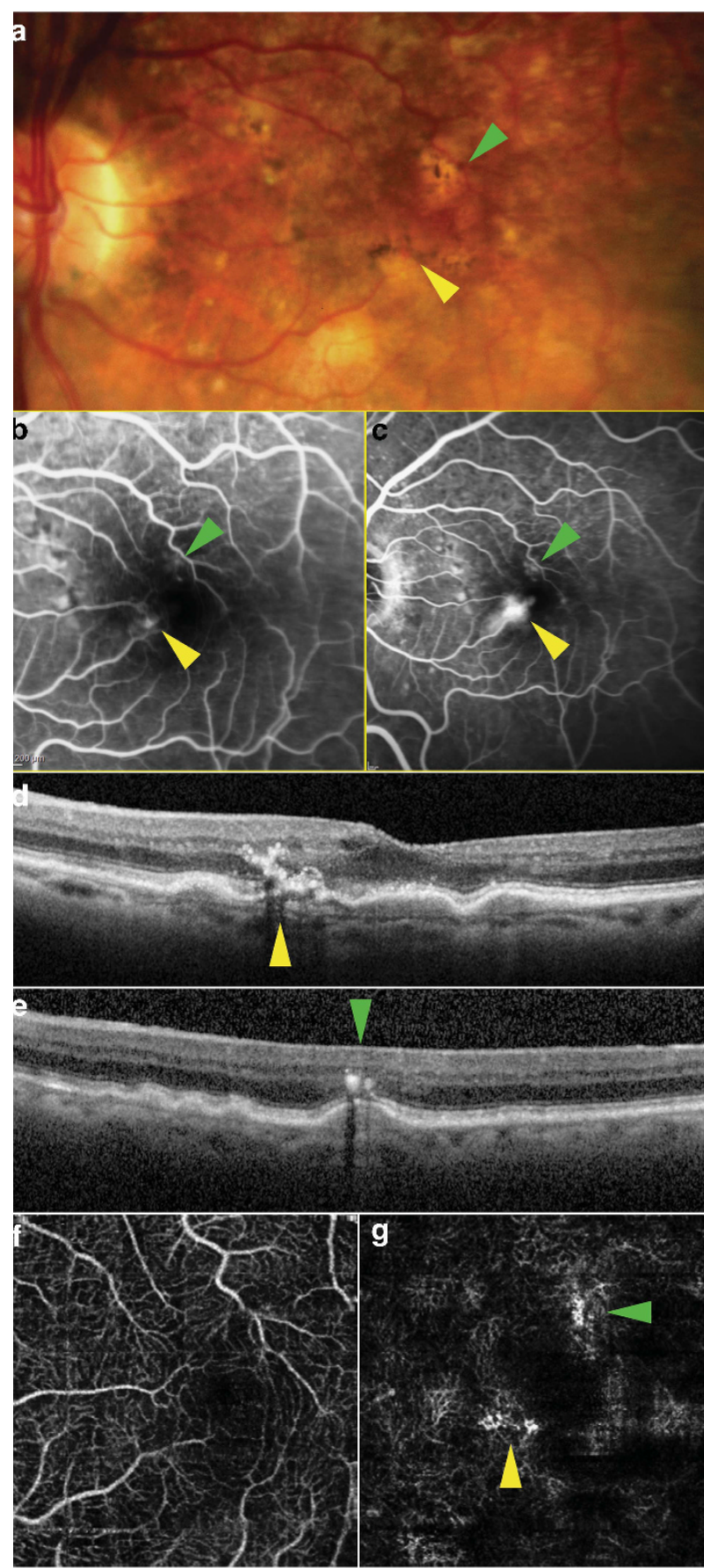

Figure 1 Multimodal imaging of the left eye of patient 1 showing two type $3 \mathrm{NV}$ lesions bordering the foveal avascular zone. Yellow arrows: high-flow lesion; green arrows: second, low-flow lesion. (a) Color photograph; (b, c) mid- and latevenous phases of fluorescein angiogram; $(d, e)$ SD-OCT through high- and low-flow lesions, respectively; (f, g) en face OCT-A segments of superficial and deep capillary plexus, respectively.

was processed by Topcon using a proprietary angiography algorithm. The resulting images revealed a hyperintense flow signal at the inferior border of the foveal avascular zone correlating with the location of the type $3 \mathrm{NV}$ (Figure 2e). The patient began treatment with intravitreal ranibizumab in the right eye on a monthly regimen. After two consecutive treatments, SD-OCT showed a reduction in the retinal thickness with partial resolution of the retinal cyst and intraretinal hemorrhage. Repeat OCT-A showed a reduction in the brightness of the type 3 lesion (Figure 2f); it also revealed a region of supranormal flow, inferotemporal to the main lesion (Figure 2f, black arrow).

\section{Discussion}

Type $3 \mathrm{NV}$ may be difficult to diagnose in the early stages, when it may manifest as intraretinal hyperreflective features on OCT, without retinal thickening or obvious RPE disruption; 5 serial FA may be necessary for early detection.

OCT-A relies on the detection of changes-in amplitude, phase, or other physical characteristics of the reflected light-that result from the interaction with the components of the flowing blood. ${ }^{6}$ These are rendered as two-dimensional en face maps in which the pixel brightness represents blood flow. ${ }^{7}$ The relative absence of stationary or time-static structures from OCT-A maps distinguishes these images from those rendered by conventional en face OCT.

Our first patient had two foci of type $3 \mathrm{NV}$. The lesion at the superior border of the foveal avascular zone was seen on SD-OCT but was only detected weakly by FA. OCT-A demonstrated both the lesions, one with a stronger flow signal than the other (the inferior, brighter lesion saturating the detection capabilities of the device). En face segmentation enhanced the sensitivity of OCT-A to detect these lesions, by excluding the flow signals from the inner retinal capillaries. Segmentation also localized the lesions to the deep capillary plexus, consistent with type $3 \mathrm{NV}$.

In the second patient, OCT-A demonstrated a single type $3 \mathrm{NV}$ lesion in the deep capillary plexus,

communicating with a sub-RPE component. Sequential OCT-A suggested a reduction in the intralesional flow as a result of the treatment with 2 anti-VEGF injections as represented by a reduction in brightness. It also revealed a complex of ectatic capillaries, inferior to the main lesion, with supranormal flow, not detectable by FA or ICGA in our patient. Telangiectasis adjacent to type $3 \mathrm{NV}$ has recently been described as an early feature of type $3 \mathrm{NV} .{ }^{8}$

The technology to perform OCT-A in the clinical setting is now commercially available. OCT-A detects, and may quantify, flow in early or partially treated type $3 \mathrm{NV}$ lesions, and has the potential to increase our understanding of the pathogenesis. To the best of our knowledge, this is the first published report of OCT-A of 


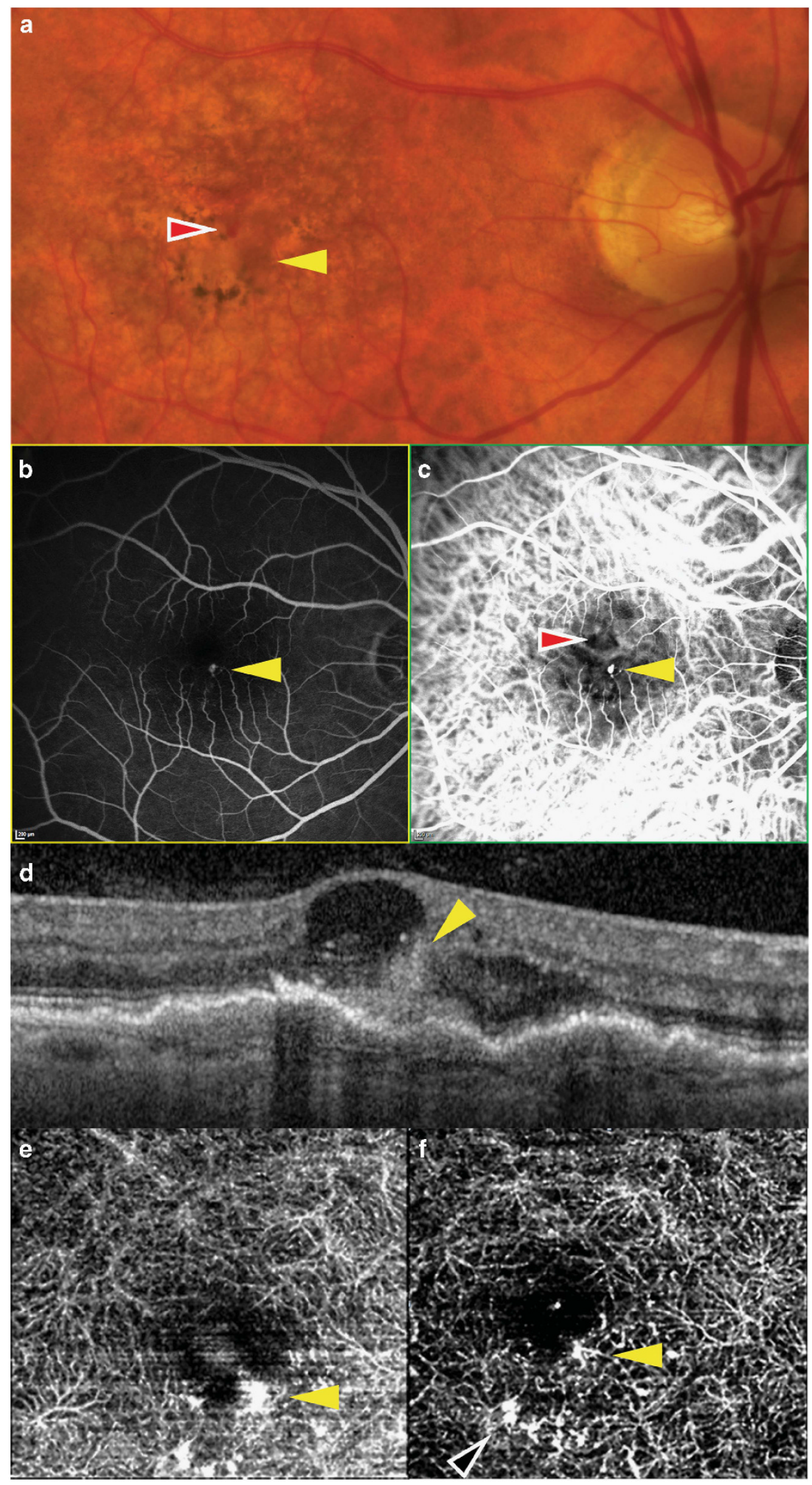

Figure 2 Multimodal imaging of the right eye of patient 2 showing a type $3 \mathrm{NV}$ lesion (yellow arrow) at the inferior border of the foveal avascular zone, and accompanying intraretinal hemorrhage that is adjacent and superior (red arrow). (a) Color photograph; (b) fluorescein angiogram; (c) indocyanine green angiogram; (d) SD-OCT showing a hyper-reflective intraretinal lesion communicating with a localized pigment epithelial detachment at its outer aspect and an adjacent hemorrhage within a cystic space at its inner aspect; (e) baseline OCT angiogram; (f) OCT angiogram after two intravitreal treatments. Supranormal flow is seen in a region of the capillary plexus inferior to the type 3 lesion (black arrow). 
type $3 \mathrm{NV}$. Larger studies are needed to determine the sensitivity and specificity of OCT-A in the early diagnosis of type $3 \mathrm{NV}$, and its reliability in determining lesion activity, through quantification of microvascular flow.

\section{Summary}

What was known before

- Type $3 \mathrm{NV}$ is well described on the basis of clinical findings and multimodal imaging including FA and crosssectional OCT.

What this study adds

- Our cases show that OCT-A is capable of demonstrating flow in early type $3 \mathrm{NV}$ (that is only weakly detectable on FA), and that OCT-A is capable of quantifying a reduction in flow following intravitreal therapy.

\section{Conflict of interest}

$\mathrm{KBF}$ is a consultant for Heidelberg Engineering.

\section{Acknowledgements}

This study was funded by LuEsther T Mertz Retinal Research Center, Manhattan Eye, Ear and Throat Hospital, New York and the Macula Foundation Inc., New York, NY, USA. The funding organization had no role in the design or conduct of this research. Swept source OCT imaging with the Topcon DRI OCT-1 was performed under an IRB approved protocol with informed consent from the patient. This work is original and has not been presented or published elsewhere.

\section{References}

1 Hartnett ME, Weiter JJ, Garsd A, Jalkh AE. Classification of retinal pigment epithelial detachments associated with drusen. Graefes Arch Clin Exp Ophthalmol 1992; 230(1): 11-19.

2 Kuhn D, Meunier I, Soubrane G, Coscas G. Imaging of chorioretinal anastomoses in vascularized retinal pigment epithelium detachments. Arch Ophthalmol 1995; 113(11): 1392-1398.

3 Yannuzzi LA, Negrao S, Iida T, Carvalho C, Rodriguez-Coleman $\mathrm{H}$, Slakter J et al. Retinal angiomatous proliferation in age-related macular degeneration. Retina 2001; 21(5): 416-434.

4 Freund KB, Ho IV, Barbazetto IA, Koizumi H, Laud K, Ferrara D et al. Type 3 neovascularization: the expanded spectrum of retinal angiomatous proliferation. Retina 2008; 28 (2): 201-211.

5 Nagiel A, Sarraf D, Sadda SR, Spaide RF, Jung J, Bhavsar K et al. Type 3 Neovascularization: evolution, association with pigment epithelial detachment, and treatment response as revealed by spectral-domain optical coherence tomography. Retina 2015; e-pub ahead of print 2 February 2015.

6 Jia Y, Tan O, Tokayer J, Potsaid B, Wang Y, Liu JJ et al. Split-spectrum amplitude-decorrelation angiography with optical coherence tomography. Opt Express 2012; 20(4): 4710-4725.

7 Tokayer J, Jia Y, Dhalla AH, Huang D. Blood flow velocity quantification using split-spectrum amplitude-decorrelation angiography with optical coherence tomography. Biomed Opt Express 2013; 4(10): 1909-1924.

8 Querques G, Souied EH, Freund KB. How has highresolution imaging refined our understanding of the vasogenic process in type 3 neovascularization? Retina 2015; e-pub ahead of print 23 January 2015. 